\title{
Radix Angelicae Sinensis/Radix Astragali Herbal Supplement
}

\author{
National Cancer Institute
}

\section{Source}

National Cancer Institute. Radix Angelicae Sinensis/Radix Astragali Herbal Supplement. NCl Thesaurus. Code C113297.

A traditional Chinese medicine comprising of Radix Angelicae Sinensis (RAS) and Radix Astrag ali (RA), with potential anti-inflammatory, immunostimulatory, neuroprotective, anti-hepatotoxic and antineoplastic activities. The main chemical constituents of RAS include ferulic acid, Z-ligustilide, butylidenephthalide and various polysaccharides. RA is the dried root of Astrag alus membranaceus with primary constituents such polysaccharides, triterpenoids as well as isoflavones. Though their mechanisms of action remain largely elusive, Radix Angelicae Sinensis/Radix Astragali herbal supplements are commonly used for the treatment of various health conditions affecting women including premenstrual syndrome, dysmenorrhea, pelvic pain, recovery from childbirth and menopausal symptoms. These agents are also used for alleviating constipation, preventing and treating anemia and allergic attacks, and for the management of hypertension, joint pain and ulcers. 